\title{
POWER GENERALIZED WEIBULL DISTRIBUTION BASED ON ORDER STATISTICS
}

\author{
DEVENDRA KUMAR* \\ Department of Statistics, Central University of Haryana, India \\ Email: devendrastats@gmai.com \\ SANKU DEY \\ Department of Statistics, St. Anthony's College Shillong, Meghalaya, India \\ Email:sankud66@gmail.com
}

\section{SUMMARY}

\begin{abstract}
In this article, we establish recurrence relations for the single and product moments of order statistics from the power generalized Weibull (PGW) distribution due to Bagdonovacius and Nikulin (2002). These recurrence relations enable computation of the means, variances and covariances of all order statistics for all sample sizes in a simple and efficient manner. By using these relations, we have obtained the means, variances and covariances of order statistics from samples of sizes up to 5 for various values of the shape and scale parameters and present them in figures.
\end{abstract}

Keywords and phrases: Characterization, Recurrence relations, Single and product moments

AMS Classification: 62G30, 65Q30

\section{Introduction}

The two-parameter Weibull distribution is a very popular distribution that has been extensively used over the past decades for modeling data in reliability, engineering and biological studies. Owing to its flexibilities, it can take the form of either the exponential distribution, the Rayleigh distribution or can be skewed either positively or negatively. However, in cases where the hazard rates are bathtub or unimodal shapes, the Weibull distribution does not provide a reasonable parametric fit. To add more flexibility to Weibull distribution, many researchers developed many generalizations of the Weibull distribution by adding new parameters. However, with the increased number of parameters in the modified or extended version of the model, the forms of the survival and hazard functions have become more and more complicated and the estimation problems have become a challenging task (Bebbington et al., 2007; Mudholkar and Srivastava, 1993; Ghitany et al., 2005; Wahed et al., 2009; Cordeiro et al., 2010; Silva et al., 2010; Ristić and Balakrishnan, 2012). The power generalized Weibull (PGW) distribution is another extension of the Weibull distribution

^ Corresponding author

(C) Institute of Statistical Research and Training (ISRT), University of Dhaka, Dhaka 1000, Bangladesh. 
which was first proposed by Bagdonovac̃ius and Nikulin in (2002). Based on parameter values, the hazard function of PGW distribution can be constant, monotone (increasing and decreasing), bathtub shaped and upside down bathtub shaped. For more details about this extension, we refer the readers to Bagdonavic̃ius et al. (2006). Besides, it is a right skewed heavy tailed distribution which is not very common in life time model. The PGW family can be used as a possible alternative to the Exponentiated Weibull family for modeling lifetime data (Nikulin and Haghighi, 2009).

Bagdonovic̃ius and Nikulin (2002) proposed the PGW distribution, which was further studied by Nikulin and Haghighi (2006), Alloyarova et al. (2007), Nikulin and Haghighi (2009), Bagdonavičius and Nikulin (2011), Voinov et al. (2013) and Mohie EL-Din et al. (2015). Nikulin and Haghighi (2006) proposed chi-squared type statistic to test the validity of the Power Generalized Weibull family based on Head-and-Neck cancer censored data. Alloyarova et al. (2007) constructed the Hsuan-Robson-Mirvaliev (HRM)(Hsuan and Robson, 1976; Mirvaliev, 2001) statistic for testing the hypothesis based on moment-type estimators and investigated its properties. Nikulin and Haghighi (2009) obtained maximum likelihood estimates of the parameters and the flexibility of the model was shown by using Efron's (1988) head-and-neck cancer clinical trial data. Bagdonavičius and Nikulin (2011) proposed chi-squared goodness of fit test for right censored data. Voinov et al. (2013) constructed modified chi-squared tests based on MLEs. Further, they studied power of the tests against exponentiated Weibull, three-parameter Weibull, and generalized Weibull distributions using Monte Carlo simulations. Recently, Mohie EL-Din et al. (2015) obtained maximum likelihood and Bayes estimates based on progressive censoring using step-stress partially accelerated life tests. Further, they obtained the approximate and the bootstrap confidence intervals of the estimators.

Order statistics and functions of these statistics play an important role in a wide range of theoretical and practical problems such as characterization of probability distributions and goodness-offit tests, entropy estimation, analysis of censored samples, reliability analysis, quality control and strength of materials (see Arnold et al. (1992) and David and Nagaraja (2003) and the references therein for more details). The practicability of moments of order statistics can be seen in many areas such as quality control testing, reliability, etc. For instance, when the reliability of an item or product is high, the duration of the failed items will be high which in turn will make the product expensive, both in terms of time and money. This prevents a practitioner from knowing enough about the product in a relatively short time. Therefore, a practitioner needs to predict the failure of future items based on the times of a few early failures. These predictions are often based on moments of order statistics.

The recurrence relations and identities have great significance because they are useful in reducing the number of operations necessary to obtain a general form for the function under consideration and they reduce the amount of direct computation, time and labour. This concept is well-established in the statistical literature, see Arnold et al. (1992). Furthermore, they are used in characterizing distributions, which is an important area, permitting the identification of population distribution from the properties of the sample. Kumar $(2013,2014,2015)$ have established recurrence relations for marginal and joint moment generating functions of lower generalized order statistics and generalized order statistics from Marshall-Olkin extended logistic, extended type II generalized logistic and 
type II exponentiated log-logistic distribution respectively. Balakrishnan et al. (2015) established some recurrence relations for single and product moments of order statistics of the complementary exponential-geometric distribution. Recently, Kumar et al. (2016) have established the relations for order statistics from extended exponential distribution. The computation of moments of order statistics is a challenging task for many distributions. For this reason, recursive computational methods are often sought.

A random variable $X$ has the PGW distribution with parameters $\alpha, \beta$ and $\sigma$, say $P G W$, if its probability density function $(p d f)$ is

$$
f(x)=\frac{\alpha}{\beta \sigma^{\alpha}} x^{\alpha-1}\left[1+\left(\frac{x}{\sigma}\right)^{\alpha}\right]^{\frac{1}{\beta}-1} e^{1-\left[1+\left(\frac{x}{\sigma}\right)^{\alpha}\right]^{\frac{1}{\beta}}} ; \quad x>0, \alpha, \beta, \sigma>0
$$

the corresponding cumulative distribution function $(c d f)$ is

$$
F(x)=1-e^{1-\left[1+\left(\frac{x}{\sigma}\right)^{\alpha}\right]^{\frac{1}{\beta}}} ; \quad x>0, \quad \alpha, \beta, \sigma>0
$$

The hazard function is given by

$$
h(x)=\frac{f(x)}{1-F(x)}=\frac{\alpha}{\beta \sigma^{\alpha}} x^{\alpha-1}\left[1+\left(\frac{x}{\sigma}\right)^{\alpha}\right]^{\frac{1}{\beta}-1} \cdot x>0 .
$$

If $\beta$ is real and non-integer and $|x|<1$, then (Gradshteyn and Ryzhik 2007; p. 25)

$$
(1+x)^{\beta}=\sum_{k=0}^{\infty}\left(\begin{array}{l}
\beta \\
k
\end{array}\right) x^{k} .
$$

One can observe from (1.1) and (1.2) that

$$
f(x)=\frac{\alpha}{\beta \sigma^{\alpha}}\left[1+\left(\frac{x}{\sigma}\right)^{\alpha}\right]^{\frac{1}{\beta}-1} \bar{F}(x)=\frac{\alpha}{\beta \sigma^{\alpha}} \sum_{u=0}^{\infty}\left(\begin{array}{c}
\frac{1}{\beta}-1 \\
u
\end{array}\right) \frac{x^{\alpha(u+1)-1}}{\sigma^{\alpha u}} \bar{F}(x),
$$

where $\bar{F}(x)=1-F(x)$. Now, the relation in (1.4) will be exploited to derive recurrence relations for the moments of order statistics for the PGW distribution.

The rest of the paper proceeds as follows: In Section 2, we describe briefly the preliminaries of order statistics. In Section 3, we derive explicit expressions and recurrence relations for single and product moments of order statistics. In Section 4, we provide the characterization of PGW distribution based on conditional moments of order statistics. In Section 5, we describe the computational procedure that will produce all the means, variances and covariances of all order statistics for all sample sizes $\mathrm{n}$. The means, variances and covariances of order statistics are presented in Figures 1-3. Finally, in Section 6, we make some concluding remarks.

\section{Order Statistics and Preliminaries}

Let $X_{1}, \ldots, X_{n}$ be a random sample of size $n$ from the PGW distribution in Equation (1.1), and let $X_{1: n} \leq \cdots \leq X_{n: n}$ denote the corresponding order statistics. Then the pdf of $X_{r: n}, 1 \leq r \leq n$, is 
(Arnold et al., 1992; David and Nagaraja, 2003)

$$
f_{r: n}(x)=C_{r: n}[F(x)]^{r-1}[1-F(x)]^{n-r} f(x), \quad 0<x<\infty,
$$

where $f(x)$ and $F(x)$ are as in Equations (1.1) and (1.2), respectively. The joint pdf of $X_{r: n}$ and $X_{s: n},(1 \leq r<s \leq n)$, is (Arnold et al., 1992; David and Nagaraja, 2003)

$$
f_{r, s: n}(x, y)=C_{r, s: n}[F(x)]^{r-1}[F(y)-F(x)]^{s-r-1}[1-F(y)]^{n-s} f(x) f(y), 0<x<y<\infty,
$$

where $C_{r: n}=[B(r, n-r+1)]^{-1}, C_{r, s: n}=[B(r, s-r, n-s+1)]^{-1}, B(a, b)=\Gamma(a) \Gamma(b) / \Gamma(a+$ $b)$ denotes the complete beta function and $B(a, b, c)=\Gamma(a) \Gamma(b) \Gamma(c) / \Gamma(a+b+c),(a, b, c>0)$ denotes the generalized beta function.

\section{Relations for Single and Product Moments of Order Statistics}

In this section, we derive explicit expressions and recurrence relations for single and product moments of order statistics from the PGW distribution.

\subsection{Relations for Single Moments}

We shall first establish explicit expressions for $j$ th single moments of the $r$ th order statistics, $E\left(X_{r: n}^{(j)}\right)=$ $\mu_{r: n}^{(j)}$. Theorem 1 gives an explicit expression for $1 \leq r \leq n$ and $j=0,1,2, \ldots$ Theorem 2 gives an explicit expression for $1 \leq r \leq n$ and $j$ a negative integer.

Theorem 1. For the $P G W$ distribution given in (1.1) and for $1 \leq r \leq n$ and $j=0,1,2, \ldots$,

$$
\mu_{r: n}^{(j)}=\sigma^{j} C_{r: n} \sum_{u=0}^{r-1} \sum_{k=0}^{j / \alpha}(-1)^{u+\frac{j}{\alpha}-k}\left(\begin{array}{c}
r-1 \\
u
\end{array}\right)\left(\begin{array}{c}
j / \alpha \\
k
\end{array}\right) \frac{e^{n+u-r+1} \Gamma(\beta k+1, n+u-r+1)}{(n+u-r+1)^{\beta k+1}},
$$

where $\Gamma(a, x)$ denotes the incomplete gamma function defined by $\Gamma(a, x)=\int_{x}^{\infty} t^{a-1} e^{t} d t$.

Proof. Using (2.1), we have

$$
\begin{aligned}
\mu_{r: n}^{(j)} & =C_{r: n} \int_{0}^{\infty} x^{j}[F(x)]^{r-1}[1-F(x)]^{n-r} f(x) d x \\
& =C_{r: n} \sum_{u=0}^{r-1}(-1)^{u}\left(\begin{array}{c}
r-1 \\
u
\end{array}\right) \int_{0}^{\infty} x^{j}[1-F(x)]^{u+n-r} f(x) d x \\
& =\frac{\alpha}{\beta \sigma^{\alpha}} C_{r: n} \sum_{u=0}^{r-1}(-1)^{u}\left(\begin{array}{c}
r-1 \\
u
\end{array}\right) e^{n-r+u+1} \int_{0}^{\infty} x^{j+\alpha-1}\left[1+\left(\frac{x}{\sigma}\right)^{\alpha}\right]^{\frac{1}{\beta}-1} e^{-(n-r+u+1)\left\{1+\left(\frac{x}{\sigma}\right)^{\alpha}\right\}^{\frac{1}{\beta}}} d x \\
& =C_{r: n} \sigma^{j} \sum_{u=0}^{r-1}(-1)^{u}\left(\begin{array}{c}
r-1 \\
u
\end{array}\right) \frac{e^{n-r+u+1}}{(n-r+u+1)} \int_{n-r+u+1}^{\infty}\left[\left(\frac{y}{n-r+u+1}\right)^{\beta}-1\right]^{j / \alpha} e^{-y} d y \\
& =C_{r: n} \sigma^{j} \sum_{u=0}^{r-1} \sum_{k=0}^{j / \alpha}(-1)^{u-k+j / \alpha} \frac{\left(\begin{array}{c}
r-1 \\
u
\end{array}\right)\left(\begin{array}{c}
j / \alpha \\
k
\end{array}\right) e^{n-r+u+1}}{(n-r+u+1)^{\beta k+1}} \int_{n-r+u+1}^{\infty} y^{\beta k} e^{-y} d y,
\end{aligned}
$$


where $y=(n-r+u+1)\left[1+\left(\frac{x}{\sigma}\right)^{\alpha}\right]^{\frac{1}{\beta}}$. The result follows from the definition of the incomplete gamma function.

In particular, the mean and the variance order statistic are

$$
\mu_{r: n}^{(1)}=\sigma C_{r: n} \sum_{u=0}^{r-1} \sum_{k=0}^{1 / \alpha}(-1)^{u+\frac{1}{\alpha}-k}\left(\begin{array}{c}
r-1 \\
u
\end{array}\right)\left(\begin{array}{c}
1 / \alpha \\
k
\end{array}\right) \frac{e^{n+u-r+1} \Gamma(\beta k+1, n+u-r+1)}{(n+u-r+1)^{\beta k+1}},
$$

and

$$
\begin{aligned}
\sigma_{r: n}^{2} & =\mu_{r: n}^{(2)}-\left(\mu_{r: n}\right)^{2} \\
& =\sigma^{2} C_{r: n} \sum_{u=0}^{r-1} \sum_{k=0}^{2 / \alpha} \frac{(-1)^{u+\frac{2}{\alpha}-k}\left(\begin{array}{c}
r-1 \\
u
\end{array}\right)\left(\begin{array}{c}
2 / \alpha \\
k
\end{array}\right) e^{n+u-r+1}}{(n+u-r+1)^{\beta k+1}} \Gamma(\beta k+1, n+u-r+1)-\left(\mu_{r: n}\right)^{2},
\end{aligned}
$$

respectively.

Theorem 2. For the PGW distribution given in (1.1) and for $1 \leq r \leq n$ and $j$ a negative integer,

$$
\mu_{r: n}^{(j)}=\frac{C_{r: n}}{\sigma^{j}} \sum_{u=0}^{r-1} \sum_{k=0}^{\infty}(-1)^{u+\frac{j}{\alpha}-k}\left(\begin{array}{c}
r-1 \\
u
\end{array}\right)\left(\begin{array}{c}
j / \alpha \\
k
\end{array}\right) \frac{e^{n+u-r+1} \Gamma(\beta k+1, n+u-r+1)}{(n+u-r+1)^{\beta k+1}} .
$$

where $\Gamma(a, x)$ denotes the incomplete gamma function.

Proof. Similar to the proof of Theorem 1.

Theorem 3 establishes a recurrence relation for $\mu_{r: n}^{(j)}$. This result holds for positive as well as negative $j$.

Theorem 3. For the PGW distribution given in (1.1) and for $1 \leq r \leq n$,

$\mu_{r: n}^{(j)}=\frac{\alpha}{\beta} \sum_{u=0}^{\frac{1}{\beta}-1}\left(\begin{array}{c}\frac{1}{\beta}-1 \\ u\end{array}\right) \frac{\sigma^{-\alpha(u+1)}}{j+\alpha(u+1)}\left[(n-r+1) \mu_{r: n}^{(j+\alpha(u+1))}-\frac{(r-1) C_{r ; n}}{C_{r-1 ; n}} \mu_{r-1: n}^{(j+\alpha(u+1))}\right]$.

Throughout, we follow the conventions that $\mu_{0: m}^{(j)}=0$ for $m \geq 1$ and $\mu_{r: m}^{(0)}=1$ for $1 \leq r \leq m$.

Proof. For $1 \leq r \leq n$, we have from (1.4) and (2.1)

$$
\mu_{r: n}^{j}=C_{r: n} \sum_{u=0}^{\frac{1}{\beta}-1}\left(\begin{array}{c}
\frac{1}{\beta}-1 \\
u
\end{array}\right) \frac{\alpha}{\beta \sigma^{\alpha(u+1)}} \int_{0}^{\infty} x^{j+\alpha(u+1)-1}[F(x)]^{r-1}[1-F(x)]^{n-r+1} d x .
$$


By integrating by parts, we obtain

$$
\begin{aligned}
\mu_{r: n}^{j}= & C_{r: n} \sum_{u=0}^{\frac{1}{\beta}-1}\left(\begin{array}{c}
\frac{1}{\beta}-1 \\
u
\end{array}\right) \frac{\alpha}{\beta \sigma^{\alpha(u+1)}}\left\{\frac{n-r+1}{j+\alpha(u+1)} \int_{0}^{\infty} x^{j+\alpha(u+1)}[F(x)]^{r-1}[1-F(x)]^{n-r} f(x) d x\right. \\
& \left.-\frac{r-1}{j+\alpha(u+1)} \int_{0}^{\infty} x^{j+\alpha(u+1)}[F(x)]^{r-2}[1-F(x)]^{n-r+1} f(x) d x\right\} \\
= & \sum_{u=0}^{\frac{1}{\beta}-1}\left(\begin{array}{c}
\frac{1}{\beta}-1 \\
u
\end{array}\right) \frac{\alpha}{\beta \sigma^{\alpha(u+1)}}\left\{\frac{n-r+1}{j+\alpha(u+1)} \mu_{r: n}^{j+\alpha(u+1)}-\frac{(r-1) C_{r: n}}{j+\alpha(u+1) C_{r-1: n}} \mu_{r-1: n}^{j+\alpha(u+1)}\right\} .
\end{aligned}
$$

The result follows.

In particular, upon setting $r=1$ in Theorem 3, we deduce the following result.

Corollary 3.1. For $n \geq 1$ and $j=0,1,2, \ldots$,

$$
\mu_{1: n}^{(j)}=n \sum_{u=0}^{\frac{1}{\beta}-1}\left(\begin{array}{c}
\frac{1}{\beta}-1 \\
u
\end{array}\right) \frac{\alpha}{\beta \sigma^{\alpha(u+1)}[j+\alpha(u+1)]} \mu_{1: n}^{(j+\alpha(u+1))} .
$$

\subsection{Relations for Product Moments}

We shall first establish explicit expressions for $i$ th and $j$ th product moment of $r$ th and $s$ th order statistics, $E\left(X_{r, s: n}^{(i, j)}\right)=\mu_{r, s: n}^{(i, j)}$. Theorem 4 gives an explicit expression for $1 \leq r<s \leq n$ and $i, j=0,1,2, \ldots$. Theorem 5 gives an explicit expression for $1 \leq r<s \leq n, i=0,1,2, \ldots$ and $j$ a negative integer. Theorem 6 gives an explicit expression for $1 \leq r<s \leq n, j=0,1,2, \ldots$ and $i$ a negative integer. Theorem 7 gives an explicit expression for $1 \leq r<s \leq n$ and both $i$ and $j$ negative integers.

Theorem 4. For the PGW distribution given in (1.1) and for $1 \leq r<s \leq n$ and $i, j=0,1,2, \ldots$,

$$
\begin{aligned}
\mu_{r, s: n}^{(i, j)}= & C_{r, s: n} \sigma^{i+j} \sum_{k=0}^{r-1} \sum_{l=0}^{s-r-1} \sum_{p=0}^{j / \alpha} \sum_{q=0}^{i / \alpha}(-1)^{k+l+\left(\frac{i+j}{\alpha}\right)-p-q}\left(\begin{array}{c}
r-1 \\
k
\end{array}\right)\left(\begin{array}{c}
s-r-1 \\
l
\end{array}\right) \\
& \times\left(\begin{array}{c}
j / \alpha \\
p
\end{array}\right)\left(\begin{array}{c}
i / \alpha \\
q
\end{array}\right) \frac{e^{n-r+k+1} \Gamma[\beta(p+q)+2]}{(n-s+l+1)^{\beta p+1}(k+s-r-l)^{\beta q+1}} \\
& \times \frac{1}{2^{\beta(p+q)+2}(\beta q+1)}{ }^{2} F_{1}(1, \beta(p+q)+2 ; \beta q+2 ; 1 / 2),
\end{aligned}
$$

where ${ }_{2} F_{1}(a, b ; c ; x)$ denotes the Gauss hyper-geometric function defined by

$$
{ }_{2} F_{1}(a, b ; c ; x)=\sum_{k=0}^{\infty} \frac{(a)_{k}(b)_{k}}{(c)_{k}} \frac{x^{k}}{k !},
$$

where $(e)_{k}=e(e+1) \cdots(e+k-1)$ denotes the ascending factorial. 
Proof. From (2.2), we have

$$
\begin{aligned}
& \mu_{r, s: n}^{(i, j)}=C_{r, s: n} \int_{0}^{\infty} \int_{x}^{\infty} x^{i} y^{j}[F(x)]^{r-1}[F(y)-F(x)]^{s-r-1}[1-F(y)]^{n-s} f(x) f(y) d y d x \\
& =C_{r, s: n} \int_{0}^{\infty} \int_{x}^{\infty} x^{i} y^{j}[1-\{1-F(x)\}]^{r-1}[1-F(x)-\{1-F(y)\}]^{s-r-1}[1-F(y)]^{n-s} f(x) f(y) d y d x \\
& =C_{r, s: n} \sum_{k=0}^{r-1} \sum_{l=0}^{s-r-1}\left(\begin{array}{c}
r-1 \\
k
\end{array}\right)\left(\begin{array}{c}
s-r-1 \\
l
\end{array}\right)(-1)^{k+l} \\
& \times \int_{0}^{\infty} \int_{x}^{\infty} x^{i} y^{j}[1-F(x)]^{k+s-r-l-1}[1-F(y)]^{n+l-s} f(x) f(y) d y d x \\
& =\frac{\alpha^{2} C_{r, s: n}}{\beta^{2} \sigma^{2 \alpha}} \sum_{k=0}^{r-1} \sum_{l=0}^{s-r-1}\left(\begin{array}{c}
r-1 \\
k
\end{array}\right)\left(\begin{array}{c}
s-r-1 \\
l
\end{array}\right)(-1)^{k+l} e^{n-r+k+1} \\
& \times \int_{0}^{\infty} \int_{x}^{\infty} x^{i+\alpha-1} y^{j+\alpha-1}\left[1+\left(\frac{x}{\sigma}\right)^{\alpha}\right]^{\frac{1}{\beta}-1}\left[1+\left(\frac{y}{\sigma}\right)^{\alpha}\right]^{\frac{1}{\beta}-1} \\
& \times e^{-(k+s-r-l)\left\{1+\left(\frac{x}{\sigma}\right)^{\alpha}\right\}^{\frac{1}{\beta}}-(n-s+l+1)\left\{1+\left(\frac{y}{\sigma}\right)^{\alpha}\right\}^{\frac{1}{\beta}}} d y d x \\
& =\frac{\alpha \sigma^{j} C_{r, s: n}}{\beta} \sum_{k=0}^{r-1} \sum_{l=0}^{s-r-1} \sum_{p=0}^{j / \alpha}\left(\begin{array}{c}
r-1 \\
k
\end{array}\right)\left(\begin{array}{c}
s-r-1 \\
l
\end{array}\right)\left(\begin{array}{c}
j / \alpha \\
p
\end{array}\right)(-1)^{k+l+(j / \alpha)-p} \\
& \times e^{n-r+k+1} \int_{0}^{\infty} \frac{x^{i+\alpha-1}}{\sigma^{\alpha}}\left[1+\left(\frac{x}{\sigma}\right)^{\alpha}\right]^{\frac{1}{\beta}-1} e^{-(k+s-r-l)\left\{1+\left(\frac{x}{\sigma}\right)^{\alpha}\right\}^{\frac{1}{\beta}}} \\
& \times\left(\frac{1}{n-s+l+1}\right)^{\beta p+1}\left(\int_{(n-s+l+1)\left[1+\left(\frac{x}{\sigma}\right)^{\alpha}\right]^{\frac{1}{\beta}}}^{\infty} z^{\beta p} e^{-z} d z\right) d x \\
& =\frac{\alpha \sigma^{j} C_{r, s: n}}{\beta} \sum_{k=0}^{r-1} \sum_{l=0}^{s-r-1} \sum_{p=0}^{j / \alpha}\left(\begin{array}{c}
r-1 \\
k
\end{array}\right)\left(\begin{array}{c}
s-r-1 \\
l
\end{array}\right)\left(\begin{array}{c}
j / \alpha \\
p
\end{array}\right)(-1)^{k+l+(j / \alpha)-p} \\
& \times e^{n-r+k+1} \int_{0}^{\infty} \frac{x^{i+\alpha-1}}{\sigma^{\alpha}}\left[1+\left(\frac{x}{\sigma}\right)^{\alpha}\right]^{\frac{1}{\beta}-1} e^{-(k+s-r-l)\left\{1+\left(\frac{x}{\sigma}\right)^{\alpha}\right\}^{\frac{1}{\beta}}} \\
& \times\left(\frac{1}{n-s+l+1}\right)^{\beta p+1} \Gamma\left(\beta p+1, \quad\left[1+\left(\frac{x}{\sigma}\right)^{\alpha}\right]^{\frac{1}{\beta}}(k+s-r-l)\right) d x \\
& =\sigma^{i+j} C_{r, s: n} \sum_{k=0}^{r-1} \sum_{l=0}^{s-r-1} \sum_{p=0}^{j / \alpha} \sum_{q=0}^{i / \alpha}\left(\begin{array}{c}
r-1 \\
k
\end{array}\right)\left(\begin{array}{c}
s-r-1 \\
l
\end{array}\right)\left(\begin{array}{c}
j / \alpha \\
p
\end{array}\right)\left(\begin{array}{c}
i / \alpha \\
q
\end{array}\right) \\
& \times(-1)^{k+l+((i+j) / \alpha)-p-q} e^{n-r+k+1}\left(\frac{1}{n-s+l+1}\right)^{\beta p+1}\left(\frac{1}{k+s-r-l}\right)^{\beta q+1} \\
& \times \int_{k+s-r+1}^{\infty} w^{\beta q} e^{-w} \Gamma(\beta p+1, w) d w
\end{aligned}
$$

where $z=(n-s+l+1)\left[1+\left(\frac{y}{\sigma}\right)^{\alpha}\right]^{\frac{1}{\beta}}$ and $w=(k+s-r+l)\left[1+\left(\frac{x}{\sigma}\right)^{\alpha}\right]^{\frac{1}{\beta}}$. The result follows 
by using equation (6.455.1) in Gradshteyn and Ryzhik (2007) to calculate the integral in (3.4).

The proof is complete.

Theorem 5. For the PGW distribution given in (1.1) and for $1 \leq r<s \leq n$ and $i=0,1,2, \ldots$ and $j$ a negative integer,

$$
\begin{aligned}
\mu_{r, s: n}^{(i, j)} & =C_{r, s: n} \sigma^{i-j} \sum_{k=0}^{r-1} \sum_{l=0}^{s-r-1} \sum_{p=0}^{\infty} \sum_{q=0}^{i / \alpha}(-1)^{k+l+\left(\frac{i+j}{\alpha}\right)-p-q}\left(\begin{array}{c}
r-1 \\
k
\end{array}\right)\left(\begin{array}{c}
s-r-1 \\
l
\end{array}\right) \\
& \times\left(\begin{array}{c}
j / \alpha \\
p
\end{array}\right)\left(\begin{array}{c}
i / \alpha \\
q
\end{array}\right) \frac{e^{n-r+k+1} \Gamma[\beta(p+q)+2]}{(n-s+l+1)^{\beta p+1}(k+s-r-l)^{\beta q+1}} \\
& \times \frac{1}{2^{\beta(p+q)+2}(\beta q+1)}{ }^{2} F_{1}\left(1, \beta(p+q)+2 ; \beta q+2 ; \frac{1}{2}\right) .
\end{aligned}
$$

Proof. Similar to the proof of Theorem 4.

Theorem 6. For the PGW distribution given in (1.1) and for $1 \leq r<s \leq n$ and $j=0,1,2, \ldots$ and $i$ a negative integer,

$$
\begin{aligned}
\mu_{r, s: n}^{(i, j)} & =C_{r, s: n} \sigma^{j-i} \sum_{k=0}^{r-1} \sum_{l=0}^{s-r-1} \sum_{p=0}^{j / \alpha} \sum_{q=0}^{\infty}(-1)^{k+l+\left(\frac{i+j}{\alpha}\right)-p-q}\left(\begin{array}{c}
r-1 \\
k
\end{array}\right)\left(\begin{array}{c}
s-r-1 \\
l
\end{array}\right) \\
& \times\left(\begin{array}{c}
j / \alpha \\
p
\end{array}\right)\left(\begin{array}{c}
i / \alpha \\
q
\end{array}\right) \frac{e^{n-r+k+1} \Gamma[\beta(p+q)+2]}{(n-s+l+1)^{\beta p+1}(k+s-r-l)^{\beta q+1}} \\
& \times \frac{1}{2^{\beta(p+q)+2}(\beta q+1)}{ }^{2} F_{1}\left(1, \beta(p+q)+2 ; \beta q+2 ; \frac{1}{2}\right)
\end{aligned}
$$

Proof. Similar to the proof of Theorem 4.

Theorem 7. For the PGW distribution given in (1.1) and for $1 \leq r<s \leq n$ and both $i$ and $j$ negative integers,

$$
\begin{aligned}
\mu_{r, s: n}^{(i, j)} & =\frac{C_{r, s: n}}{\sigma^{i+j}} \sum_{k=0}^{r-1} \sum_{l=0}^{s-r-1} \sum_{p=0}^{\infty} \sum_{q=0}^{\infty}(-1)^{k+l+\left(\frac{i+j}{\alpha}\right)-p-q}\left(\begin{array}{c}
r-1 \\
k
\end{array}\right)\left(\begin{array}{c}
s-r-1 \\
l
\end{array}\right) \\
& \times\left(\begin{array}{c}
j / \alpha \\
p
\end{array}\right)\left(\begin{array}{c}
i / \alpha \\
q
\end{array}\right) \frac{e^{n-r+k+1} \Gamma[\beta(p+q)+2]}{(n-s+l+1)^{\beta p+1}(k+s-r-l)^{\beta q+1}} \\
& \times \frac{1}{2^{\beta(p+q)+2}(\beta q+1)}{ }^{2} F_{1}\left(1, \beta(p+q)+2 ; \beta q+2 ; \frac{1}{2}\right)
\end{aligned}
$$

Proof. Similar to the proof of Theorem 4.

Theorem 8 establishes a recurrence relation for $\mu_{r, s: n}^{(i, j)}$. This result holds for positive as well as negative values of $i$ and $j$. 
Theorem 8. For the PGW distribution given in (1.1) and for $1 \leq r<s \leq n$,

$$
\begin{aligned}
\mu_{r, s: n}^{(i, j)} & =C_{r, s: n}\left(\frac{\alpha}{\beta \sigma^{\alpha}}\right)^{2} \sum_{k=0}^{\frac{1}{\beta}-1} \sum_{l=0}^{\frac{1}{\beta}-1}\left(\begin{array}{c}
\frac{1}{\beta}-1 \\
k
\end{array}\right)\left(\begin{array}{c}
\frac{1}{\beta}-1 \\
l
\end{array}\right) \frac{1}{\sigma^{\alpha(k+l)}} \\
& \times\left[\frac{n-s+1}{j+\alpha(l+1)} I_{1}-\frac{n-s+1}{j+\alpha(l+1)} I_{2}-\frac{s-r-1}{j+\alpha(l+1)} I_{3}+\frac{s-r-1}{j+\alpha(l+1)} I_{4}\right],
\end{aligned}
$$

where

$$
\begin{aligned}
& I_{1}=-\frac{n}{i+\alpha(k+1)} \mu_{r-1, s-1: n}^{(i+\alpha(k+1), j+\alpha(l+1))}+\frac{n}{i+\alpha(k+1)} \mu_{r, s-1: n}^{(i+\alpha(k+1), j+\alpha(l+1))} \\
& I_{2}=-\frac{r}{i+\alpha(k+1)} \mu_{r, s: n}^{(i+\alpha(k+1), j+\alpha(l+1))}+\frac{n r}{i+\alpha(k+1)} \mu_{r+1, s: n}^{(i+\alpha(k+1), j+\alpha(l+1))} \\
& I_{3}=-\frac{n}{(s-r-1)[i+\alpha(k+1)]} \mu_{r-1, s-2: n-1}^{(i+\alpha(k+1), j+\alpha(l+1))}+\frac{n(n-s+1)}{(s-r-2)[i+\alpha(k+1)]} \mu_{r, s-2: n-1}^{(i+\alpha(k+1), j+\alpha(l+1))} \\
& I_{4}=-\frac{r(n-s+1)}{(s-r-1)[i+\alpha(k+1)]} \mu_{r, s-1: n}^{(i+\alpha(k+1), j+\alpha(l+1))}+\frac{r(n-s+1)}{(s-r-1)[i+\alpha(k+1)]} \mu_{r+1, s-1: n}^{(i+\alpha(k+1), j+\alpha(l+1))} .
\end{aligned}
$$

Proof. For $1 \leq r \leq n$, we have from (1.4) and (2.2)

$$
\begin{aligned}
\mu_{r, s: n}^{(i, j)} & =C_{r, s: n}\left(\frac{\alpha}{\beta \sigma^{\alpha}}\right)^{2} \sum_{k=0}^{\frac{1}{\beta}-1} \sum_{l=0}^{\frac{1}{\beta}-1}\left(\begin{array}{c}
\frac{1}{\beta}-1 \\
k
\end{array}\right)\left(\begin{array}{c}
\frac{1}{\beta}-1 \\
l
\end{array}\right) \frac{1}{\sigma^{\alpha(k+l)}} \\
& \times \int_{0}^{\infty} \int_{x}^{\infty} x^{i+\alpha(k+1)-1} y^{j+\alpha(l+1)-1}\left\{[F(x)]^{r-1}-[F(x)]^{r}\right\}[F(y)-F(x)]^{s-r-1}[1-F(y)]^{n-s+1} d y d x .
\end{aligned}
$$

By integrating by parts with respect to $y$, we obtain

$$
\begin{aligned}
\mu_{r, s: n}^{(i, j)} & =C_{r, s: n}\left(\frac{\alpha}{\beta \sigma^{\alpha}}\right)^{2} \sum_{k=0}^{\frac{1}{\beta}-1} \sum_{l=0}^{\frac{1}{\beta}-1}\left(\begin{array}{c}
\frac{1}{\beta}-1 \\
k
\end{array}\right)\left(\begin{array}{c}
\frac{1}{\beta}-1 \\
l
\end{array}\right) \frac{1}{\sigma^{\alpha(k+l)}} \\
& \times\left[\frac{n-s+1}{j+\alpha(l+1)} I_{1}-\frac{n-s+1}{j+\alpha(l+1)} I_{2}-\frac{s-r-1}{j+\alpha(l+1)} I_{3}+\frac{s-r-1}{j+\alpha(l+1)} I_{4}\right]
\end{aligned}
$$

where

$$
\begin{aligned}
& I_{1}=\int_{0}^{\infty} \int_{x}^{\infty} x^{i+\alpha(k+1)-1} y^{j+\alpha(l+1)}[F(x)]^{r-1}[F(y)-F(x)]^{s-r-1}[1-F(y)]^{n-s} f(y) d y d x \\
& I_{2}=\int_{0}^{\infty} \int_{x}^{\infty} x^{i+\alpha(k+1)-1} y^{j+\alpha(l+1)}[F(x)]^{r}[F(y)-F(x)]^{s-r-1}[1-F(y)]^{n-s} f(y) d y d x \\
& I_{3}=\int_{0}^{\infty} \int_{x}^{\infty} x^{i+\alpha(k+1)-1} y^{j+\alpha(l+1)}[F(x)]^{r-1}[F(y)-F(x)]^{s-r-2}[1-F(y)]^{n-s+1} f(y) d y d x \\
& I_{4}=\int_{0}^{\infty} \int_{x}^{\infty} x^{i+\alpha(k+1)-1} y^{j+\alpha(l+1)}[F(x)]^{r}[F(y)-F(x)]^{s-r-2}[1-F(y)]^{n-s} f(y) d y d x .
\end{aligned}
$$


By integrating by parts with respect to $x$, we can write

$$
\begin{aligned}
& I_{1}=-\frac{r-1}{i+\alpha(k+1)} J_{1}+\frac{s-r-1}{i+\alpha(k+1)} J_{2}, \\
& I_{2}=-\frac{r}{i+\alpha(k+1)} J_{3}+\frac{s-r-1}{i+\alpha(k+1)} J_{4}, \\
& I_{3}=-\frac{r-1}{i+\alpha(k+1)} J_{5}+\frac{s-r-2}{i+\alpha(k+1)} J_{6}, \\
& I_{4}=-\frac{r}{i+\alpha(k+1)} J_{7}+\frac{s-r-2}{i+\alpha(k+1)} J_{8},
\end{aligned}
$$

where

$$
\begin{aligned}
& J_{1}=\int_{0}^{\infty} \int_{x}^{\infty} x^{i+\alpha(k+1)} y^{j+\alpha(l+1)}[F(x)]^{r-2}[F(y)-F(x)]^{s-r-1}[1-F(y)]^{n-s} f(x) f(y) d y d x \\
& =\frac{C_{r, s: n}}{C_{r-1, s-1: n-1}} \mu_{r-1, s-1: n-1}^{(i+\alpha(k+1), j+\alpha(l+1))}, \\
& J_{2}=\int_{0}^{\infty} \int_{x}^{\infty} x^{i+\alpha(k+1)} y^{j+\alpha(l+1)}[F(x)]^{r-1}[F(y)-F(x)]^{s-r-2}[1-F(y)]^{n-s} f(x) f(y) d y d x \\
& =\frac{C_{r, s: n}}{C_{r, s-1: n-1}} \mu_{r, s-1: n-1}^{(i+\alpha(k+1), j+\alpha(l+1))} \\
& J_{3}=\int_{0}^{\infty} \int_{x}^{\infty} x^{i+\alpha(k+1)} y^{j+\alpha(l+1)}[F(x)]^{r-1}[F(y)-F(x)]^{s-r-1}[1-F(y)]^{n-s} f(x) f(y) d y d x \\
& =\mu_{r, s: n}^{(i+\alpha(k+1), j+\alpha(l+1))}, \\
& J_{4}=\int_{0}^{\infty} \int_{x}^{\infty} x^{i+\alpha(k+1)} y^{j+\alpha(l+1)}[F(x)]^{r}[F(y)-F(x)]^{s-r-2}[1-F(y)]^{n-s} f(x) f(y) d y d x \\
& =\frac{C_{r, s: n}}{C_{r+1, s: n}} \mu_{r+1, s: n}^{(i+\alpha(k+1), j+\alpha(l+1))} \text {, } \\
& J_{5}=\int_{0}^{\infty} \int_{x}^{\infty} x^{i+\alpha(k+1)} y^{j+\alpha(l+1)}[F(x)]^{r-2}[F(y)-F(x)]^{s-r-2}[1-F(y)]^{n-s+1} f(x) f(y) d y d x \\
& =\frac{C_{r, s: n}}{C_{r-1, s-2: n-1}} \mu_{r-1, s-2: n-1}^{(i+\alpha(k+1), j+\alpha(l+1))}, \\
& J_{6}=\int_{0}^{\infty} \int_{x}^{\infty} x^{i+\alpha(k+1)} y^{j+\alpha(l+1)}[F(x)]^{r-1}[F(y)-F(x)]^{s-r-3}[1-F(y)]^{n-s+1} f(x) f(y) d y d x \\
& =\frac{C_{r, s: n}}{C_{r, s-2: n-1}} \mu_{r, s-2: n-1}^{(i+\alpha(k+1), j+\alpha(l+1))}, \\
& J_{7}=\int_{0}^{\infty} \int_{x}^{\infty} x^{i+\alpha(k+1)} y^{j+\alpha(l+1)}[F(x)]^{r-1}[F(y)-F(x)]^{s-r-2}[1-F(y)]^{n-s+1} f(x) f(y) d y d x \\
& =\frac{C_{r, s: n}}{C_{r, s-1: n}} \mu_{r, s-1: n}^{(i+\alpha(k+1), j+\alpha(l+1))}
\end{aligned}
$$




$$
\begin{aligned}
J_{8} & =\int_{0}^{\infty} \int_{x}^{\infty} x^{i+\alpha(k+1)} y^{j+\alpha(l+1)}[F(x)]^{r}[F(y)-F(x)]^{s-r-3}[1-F(y)]^{n-s+1} f(x) f(y) d y d x \\
& =\frac{C_{r, s: n}}{C_{r+1, s-1: n}} \mu_{r+1, s-1: n}^{(i+\alpha(k+1), j+\alpha(l+1))}
\end{aligned}
$$

The result follows by combining (3.6), (3.7)-(3.10) and (3.11)-(3.18).

In particular, upon setting $s=r+1$ in Theorem 8 , we deduce the following result.

Corollary 3.2. For the PGW distribution given in (1.1) and for $1 \leq r \leq n$,

$$
\begin{aligned}
\mu_{r, r+1: n}^{(i, j)}= & C_{r, r+1: n}\left(\frac{\alpha}{\beta \sigma^{\alpha}}\right)^{2} \sum_{k=0}^{2} \sum_{l=0}^{\frac{1}{\beta}-1}\left(\begin{array}{c}
\frac{1}{\beta}-1 \\
k
\end{array}\right)\left(\begin{array}{c}
\frac{1}{\beta}-1 \\
l
\end{array}\right) \frac{1}{\sigma^{\alpha(k+l)}} \\
& \times\left[\frac{n-r}{j+\alpha(l+1)} I_{1}-\frac{n-r}{j+\alpha(l+1)} I_{2}\right]
\end{aligned}
$$

where

$$
\begin{aligned}
& I_{1}=-\frac{n}{i+\alpha(k+1)} \mu_{r-1, r: n-1}^{(i+\alpha(k+1), j+\alpha(l+1))}+\frac{n}{i+\alpha(k+1)} \mu_{r, r: n-1}^{(i+\alpha(k+1), j+\alpha(l+1))}, \\
& I_{2}=-\frac{r}{i+\alpha(k+1)} \mu_{r, r+1: n}^{(i+\alpha(k+1), j+\alpha(l+1))}+\frac{n r}{i+\alpha(k+1)} \mu_{r+1, r+1: n}^{(i+\alpha(k+1), j+\alpha(l+1))} .
\end{aligned}
$$

\section{Characterization}

In this section, we characterize the PGW distribution based on conditional moments of order statistics. Let $L(a, b)$ denote the space of all integrable functions on $(a, b)$. A sequence $\left(h_{n}\right) \subset L(a, b)$ is called complete on $L(a, b)$ if for all functions $g \in L(a, b)$ the condition

$$
\int_{a}^{b} g(x) f_{n}(x) d x=0, \quad n \in N
$$

implies $g(x)=0$ a.e. on $(a, b)$. We start with the following result of Lin (1986).

Proposition 4.1. Let $n_{0}$ be any fixed non-negative integer, $-\infty \leq a<b \leq \infty$ and $g(x) \geq 0$ an absolutely continuous function with $g^{\prime}(x) \neq 0$ a.e. on $(a, b)$. Then the sequence of functions $\left\{(g(x))^{n} e^{-g(x)}, n \geq n_{0}\right\}$ is complete in $L(a, b)$ if and only if $g(x)$ is strictly monotone on $(a, b)$.

Using the above proposition, we obtain a stronger version of Theorem 3 .

Theorem 9. Let $X$ be a non-negative random variable having an absolutely continuous cdf $F(x)$ with $F(0)=0$ and $0<F(x)<1$ for all $x>0$. Then

$$
\mu_{r: n}^{(j)}=\frac{\alpha}{\beta} \sum_{u=0}^{\frac{1}{\beta}-1}\left(\begin{array}{c}
\frac{1}{\beta}-1 \\
u
\end{array}\right) \frac{\sigma^{-\alpha(u+1)}}{j+\alpha(u+1)}\left[(n-r+1) \mu_{r: n}^{(j+\alpha(u+1))}-\frac{(r-1) C_{r ; n}}{C_{r-1 ; n}} \mu_{r-1: n}^{(j+\alpha(u+1))}\right] .
$$


if and only if

$$
F(x)=1-e^{1-\left[1+\left(\frac{x}{\sigma}\right)^{\alpha}\right]^{\frac{1}{\beta}}} ; \quad x>0, \quad \alpha, \beta, \sigma>0 .
$$

Proof. The necessary part follows immediately from equation (3.4). On the other hand if the recurrence relation in equation (4.1) is satisfied, then on using equations (1.4), we have

$$
\begin{aligned}
& C_{r: n} \int_{0}^{\infty} x^{j}[F(x)]^{r-1}[1-F(x)]^{n-r} f(x) d x= \\
& \frac{\alpha}{\beta} \sum_{u=0}^{\frac{1}{\beta}-1}\left(\begin{array}{c}
\frac{1}{\beta}-1 \\
u
\end{array}\right) \frac{1}{\sigma^{\alpha(u+1)}}\left\{\frac{(n-r+1) C_{r: n}}{j+\alpha(u+1)} \int_{0}^{\infty} x^{j+\alpha(u+1)}[F(x)]^{r-1}[1-F(x)]^{n-r} f(x) d x\right. \\
& \left.\quad-\frac{(r-1) C_{r: n}}{j+\alpha(u+1)} \int_{0}^{\infty} x^{j+\alpha(u+1)}[F(x)]^{r-2}[1-F(x)]^{n-r+1} f(x) d x\right\}
\end{aligned}
$$

Integrating the last integral on the right hand side of equation (4.2), by parts we get

$$
\begin{aligned}
& C_{r: n} \int_{0}^{\infty} x^{j}[F(x)]^{r-1}[1-F(x)]^{n-r} f(x) d x= \\
& \frac{\alpha}{\beta} \sum_{u=0}^{\frac{1}{\beta}-1}\left(\begin{array}{c}
\frac{1}{\beta}-1 \\
u
\end{array}\right) \frac{1}{\sigma^{\alpha(u+1)}}\left\{\frac{(n-r+1) C_{r: n}}{j+\alpha(u+1)} \int_{0}^{\infty} x^{j+\alpha(u+1)}[F(x)]^{r-1}[1-F(x)]^{n-r} f(x) d x\right. \\
& \quad-\frac{(n-r+1) C_{r: n}}{j+\alpha(u+1)} \int_{0}^{\infty} x^{j+\alpha(u+1)}[F(x)]^{r-1}[1-F(x)]^{n-r} f(x) d x \\
& \left.\quad+C_{r: n} \int_{0}^{\infty} x^{j+\alpha(u+1)-1}[F(x)]^{r-1}[1-F(x)]^{n-r+1} f(x) d x\right\} .
\end{aligned}
$$

which reduces to

$$
C_{r: n} \int_{0}^{\infty} x^{j}[F(x)]^{r-1}[1-F(x)]^{n-r}\left\{f(x)-\frac{\alpha}{\beta \sigma^{\alpha}} \sum_{u=0}^{\frac{1}{\beta}-1}\left(\begin{array}{c}
\frac{1}{\beta}-1 \\
u
\end{array}\right) \frac{x^{\alpha(u+1)-1}}{\sigma^{\alpha u}} \bar{F}(x)\right\} d x=0 .
$$

It now follows from Proposition 1

$$
f(x)=\frac{\alpha}{\beta \sigma^{\alpha}} \sum_{u=0}^{\frac{1}{\beta}-1}\left(\begin{array}{c}
\frac{1}{\beta}-1 \\
u
\end{array}\right) \frac{x^{\alpha(u+1)-1}}{\sigma^{\alpha u}} \bar{F}(x) \text { or } \frac{f(x)}{\bar{F}(x)}=\frac{\alpha}{\beta \sigma^{\alpha}} \sum_{u=0}^{\frac{1}{\beta}-1}\left(\begin{array}{c}
\frac{1}{\beta}-1 \\
u
\end{array}\right) \frac{x^{\alpha(u+1)-1}}{\sigma^{\alpha u}}
$$

which proves that

$$
F(x)=1-e^{1-\left[1+\left(\frac{x}{\sigma}\right)^{\alpha}\right]^{\frac{1}{\beta}}} ; \quad x>0, \quad \alpha, \beta, \sigma>0 .
$$




\section{Computational Techniques}

In this section, we describe the recursive computational algorithm that will produce all the means, variances and covariances of all order statistics for all sample sizes $\mathrm{n}$ in a simple recursive manner. We describe the computational procedure for the single and product moments separately below so that the method of computation becomes clear.

Starting with the initial values given by (3.2) and (3.3) ,the recurrence relations in Theorem 3 and Corollary 3.1 can be used recursively to compute the first two moments of all order statistics for sample sizes $n=2,3, \ldots$. From the resulting values, variances of order statistics can be readily computed. These moments were checked by using the identities

$$
\sum_{r=1}^{n} \mu_{r: n}^{(j)}=n \mu_{1: 1}^{(j)}, \quad j=1,2
$$

In Figure 1, we have presented the means of all order statistics for sample sizes $n=1(1) 5$ and $\sigma=0.25(0.25) 1.25$ and we observe that the mean of order statistics is increasing with respect to $\sigma$.
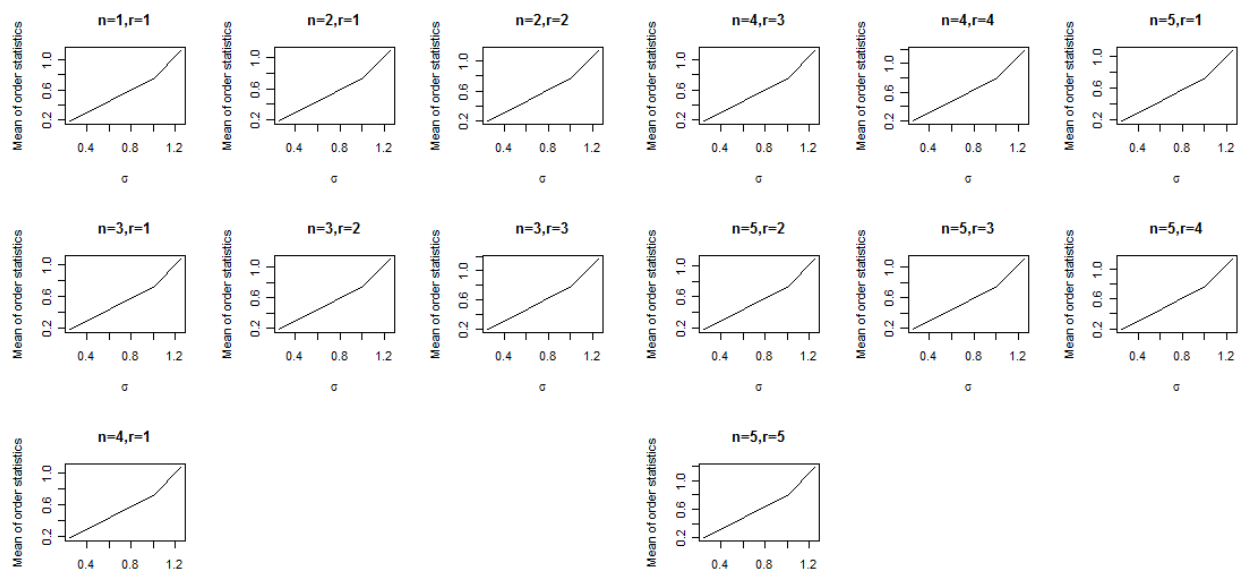

Figure 1: Mean of order statistics for $\sigma=0.25,0.5,0.75,1.0,1.25$.

For computing covariances of order statistics, all the product moments $\mu_{r, s: n}^{(1,1)}$ can be computed in a systematic manner. Starting with the initial values given by (3.1), the recurrence relations in Theorem 8 and Corollary 3.2 can be used recursively to compute $\mu_{r, s: n}^{(1,1)}$ for all $r, s$ and $n=2,3, \ldots$. The accuracy of their computation was checked by the well-known identities for any arbitrary continuous distribution

$$
\sum_{r=1}^{n} \sum_{s=1}^{n} \sigma_{r, s: n}=n \sigma_{1,1: 1}
$$


and

$$
\sum_{s=r+1}^{n} \sigma_{r, s: n}+\sum_{i=1}^{r} \sigma_{i, r+1: n}=\left(r \mu_{1: 1}^{(1)}-\sum_{i=1}^{r} \mu_{i: n}^{(1)}\right)\left(\mu_{r+1: n}^{(1)}-\mu_{r: n}^{(1)}\right), \quad 1 \leq r \leq n-1,
$$

where $\sigma_{r, s: n}=\mu_{r, s: n}^{(1,1)}-\mu_{r: n}^{(1)} \mu_{s: n}^{(1)}$ (see Joshi and Balakrishnan (1982)).
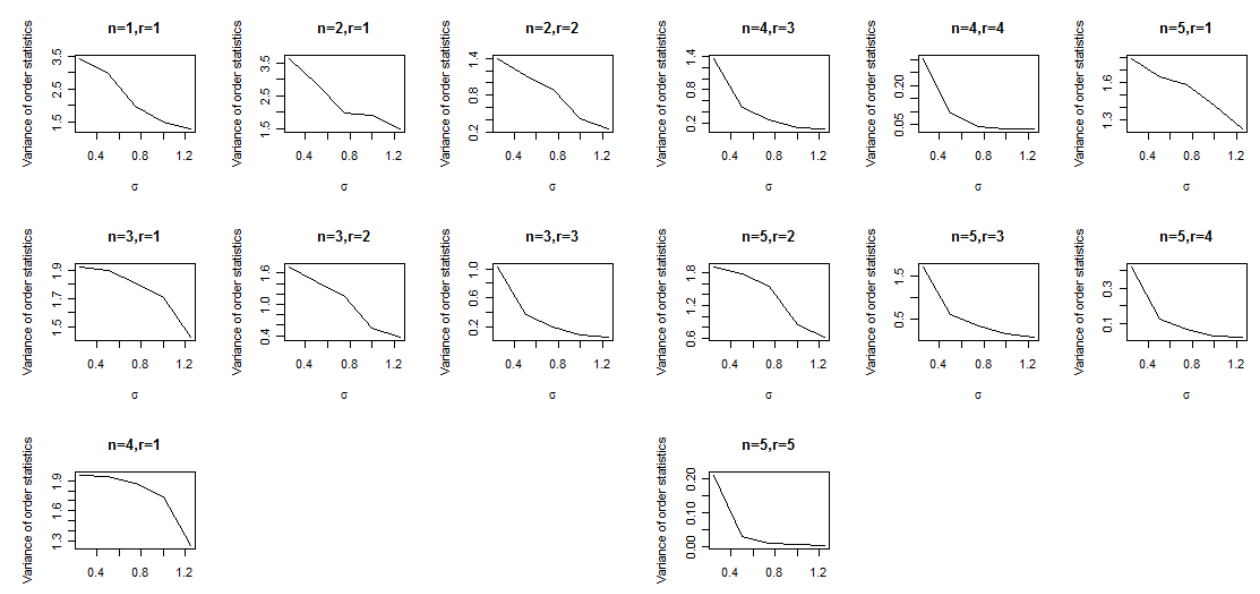

Figure 2: Variance of order statistics for $\sigma=0.25,0.5,0.75,1.0,1.25$.
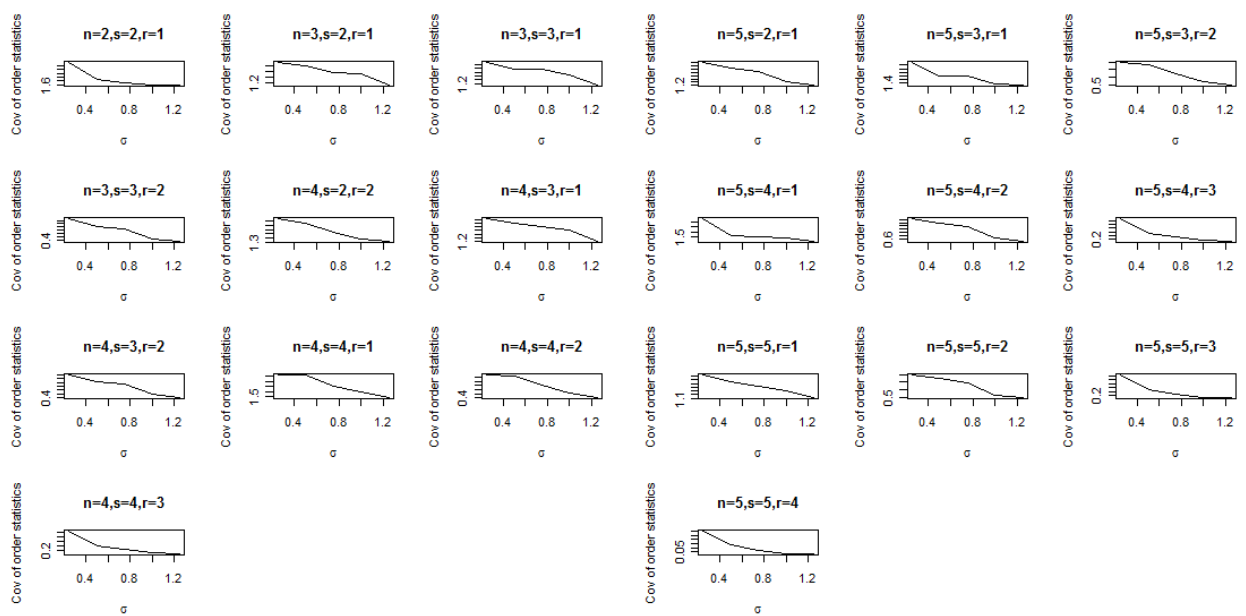

Figure 3: covariance of order statistics for $\sigma=0.25,0.5,0.75,1.0,1.25$.

The variances and covariances are presented in Figures 2 and 3 of all order statistics for sample sizes $n=1(1) 5$ and $\sigma=0.25(0.25) 1.25$ and we observe that the variances and covariances of order statistics is decreasing with respect to $\sigma$. 


\section{Discussion}

In this paper, recurrence relations for single and product moments of order statistics from PGW distribution have been derived. The recurrence relations for moments of order statistics are important because they can be helpful in reducing the amount of direct calculations needed to calculate the moments, and they can be used in a simple recursive manner to express the unknown higher order moments in terms of order statistics thus making the evaluation of higher moments easy. Also they can be used to characterize the distributions. We see that the moments of order statistics of the distribution are well behaved. This will encourage the study of the other properties of order statistics for a future research.

\section{Acknowledgements}

The authors would like to thank the reviewer and the editors for their comments which helped improve the paper.

\section{A R-code for computing the moments of order statistics}

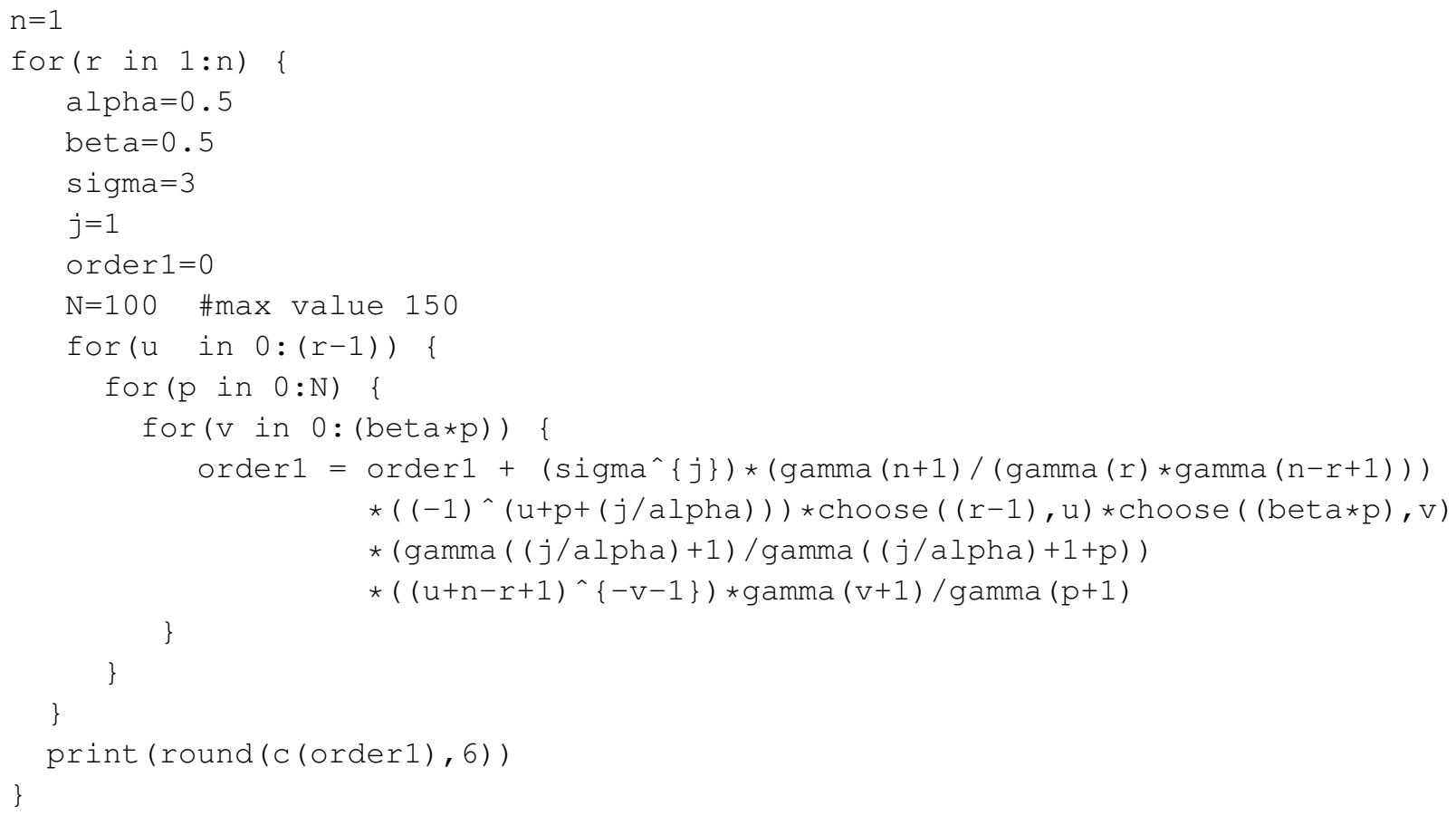




\section{References}

Arnold, B. C., Balakrishnan, N. and Nagaraja, H. N. (1992), A First Course in Order Statistics, John Wiley, New York.

Alloyarova, R., Nikulin, M., Pya, N., and Voinov, V. (2007), "The power-generalized Weibull probability distribution and its use in survival analysis", Communications in Dependability and Quality Management, 10, 5-15.

Bagdonavičius, V. and Nikulin, M. (2002), Accelerated Life Models, Chapman and Hall/CRC, Boca Raton.

Bagdonavičius, V., Clerjaud, L., and Nikulin, M. (2006) Power generalized Weibull in accelerated life testing. Preprint 0602, Statistique Mathematique et ses Applications, I.F.R. 99, Université Victor Segalen Bordeaux 2, p. 28.

Bagdonavičius, V. and Nikulin, M. (2011), "Chi-squared goodness of fit test for right censored data", International Journal of Applied Mathematics and Statistics, 24, 30-50.

Balakrishnan, N., Zhu, X. and Al-Zaharani, B. (2015), "Recursive computation of the single and product moments of order statistics for the complementary exponential-geometric distribution", Journal of Statistical Computation and Simulation, 85, 2187-2201.

Bebbington, M., Lai, C. D. and Zitikis, R. (2007), "A flexible Weibull extension”, Reliability Engineering and System Safety, 92, 719-726.

Cordeiro, G. M., Edwin, M. M. Ortega and Nadarajah, S. (2010), "The Kumaraswamy Weibull distribution with application to failure data”, J. Franklin Inst., 347, 1399-1429.

David, H. A. and Nagaraja, H. N. (2003), Order Statistics, third edition, John Wiley, New York.

Efron, B. (1988), "Logistic regression, survival analysis, and the Kaplan-Meier curve", Journal of the American Statistical Association, 83, 414-425.

Ghitany M. E., Al-Hussaini E. K. and Al-Jarallah R. A. (2005), "Marshall-Olkin extended Weibull distribution and its application to censored data", Journal of Applied Statistics, 32, 1025-1034.

Gradshteyn, I. S. and Ryzhik, I. M. (2007), Table of Integrals, Series, and Products, sixth edition, Academic Press, San Diego.

Hsuan, A. and Robson, A. D. (1976), "The $\chi^{2}$ goodness-of-fit tests with moment type estimators", Communication Statistics - Theory and Methods, A5, 1509-1519.

Joshi, P. C. and Balakrishnan, N. (1982), "Recurrence relations and identities for the product moments of order statistics", Sankhya Series B, 44, 39-49. 
Ku, P. M., Anderson, E. L. and Carper, H. J. (1972), "Some considerations in rolling fatigue evaluation", ASLE Transactions, 15, 113-129.

Kumar, D. (2013), "Relations for marginal and joint moment generating functions of MarshallOlkin extended logistic distribution based on lower generalized order statistics and characterization”, American Journal of Mathematical and Management Sciences, 32, 19-39.

Kumar, D. (2014), “On moment generating function of generalized order statistics from extended type II generalized logistic distribution", Journal of Statistical Theory and Application, 13, 135-150.

Kumar, D. (2015), "Exact moments of generalized order statistics from type II exponentiated log-logistic distribution", Hacettepe Journal of Mathematics and Statistics, 44, 715-733.

Kumar, D., Dey, S., and Nadarajah, S. (2016), "Extended exponential distribution based on order statistics", Communications in Statistics-Theory and Methods, 46, 9166-9184.

Lin, G.D. (1988), "Characterizations of distributions via relationships between two moments of order statistics”, Journal of Statistical Planning and Inference, 19, 73-80.

Mirvaliev, M. (2001), “An investigation of generalized chi-squared type statistics”. Doctoral thesis, Academy of Science of the Republic of Uzbekistan, Tashkent.

Mudholkar, G. S. and Srivastava, D. K. (1993), "Exponentiated Weibull family for analyzing bathtub failure-real data", IEEE Transaction Reliability, 42, 299-302.

Mohie EL-Din, M. M., Abu-Youssef, S. E., Nahed, A. S. A. and Abd El-Raheem, A. M. (2015), "Estimation in step-stress accelerated life tests for power generalized Weibull distribution with progressive censoring", Advances in Statistics, 1-13.

Nikulin, N. and Haghighi, F. (2009), "On the power generalized Weibull family: model for cancer censored data", Metron, LXVII, 75-86.

Nikulin, M. and Haghighi, F. (2006), "A chi-squared test for the generalized power Weibull family for the head-and-neck cancer censored data", Journal of Mathematical Sciences, 133, 1333-1341.

Ristić, M. M. and Balakrishnan, N. (2012), “The gamma-exponentiated exponential distribution”, Journal of Statistical Computation and Simulation, 82, 1191-1206.

Silva, G. O., Edwin, M. M. Ortega and Cordeiro, G. M. (2010), “The beta modified Weibull distribution", Lifetime Data Analysis, 16, 409-430.

Voinov, V., Pya, N., Shapakov, N., and Voinov, Y. (2013), "Goodness-of-fit tests for the powergeneralized weibull probability distribution", Communications in Statistics-Simulation and Computation, 42, 1003-1012. 
Wahed, A. S., Luong, T. M. and Jeong, J.-H. (2009), "A new generalization of Weibull distribution with application to a breast cancer data set", Statistics in Medicine, 28, 2077-2094.

Received: August 5, 2016

Accepted: May 22, 2017 